\title{
Metabolic Regulation, Mitochondria and the Life-Prolonging Effect of Rapamycin: A Mini-Review
}

\author{
Yong Pan Yuya Nishida Margaret Wang Eric Verdin \\ Gladstone Institute of Virology and Immunology, University of California, San Francisco, Calif., USA
}

\section{Key Words}

Target of rapamycin - Oxidative phosphorylation - Reactive oxygen species $\cdot$ Nutrient sensing $\cdot$ Mitohormesis

\begin{abstract}
The fungicide rapamycin increases lifespan in eukaryotes by interfering with the activity of a serine/threonine kinase called TOR (target of rapamycin). TOR complex 1 (TORC1) is an essential integrator of cellular nutrient cues, growth signals and cellular metabolism. Here, we review major components of TORC1, its downstream effectors and lifespan studies in various organisms involving these signaling components. In particular, we focus on the role of rapamycin in mitochondrial biogenesis, in metabolic regulation and in the control of reactive oxygen species production.
\end{abstract}

Copyright ๑ 2012 S. Karger AG, Basel

\section{Introduction: Rapamycin, TOR and Downstream Signaling Components}

Every eukaryotic organism ages, a process characterized by a progressive loss of cellular function that ultimately leads to death. Lifespan is regulated by a complex network of extrinsic and intrinsic factors. Extrinsic factors, such as nutrient levels and environmental stress ex- posure, represent a major determinant of lifespan. Organisms respond actively to extrinsic factors by triggering conserved signaling pathways that influence lifespan, as shown by extensive research in various model systems, such as mice, flies (Drosophila melanogaster) and budding yeast. The 'target of rapamycin' (TOR) pathway, which senses nutrient availability and environmental stress, has recently emerged as a major regulator of lifespan. Here, we focus on how the TOR complex 1 (TORC1) and its downstream effectors influence lifespan by regulating cellular metabolism and mitochondrial functions.

TOR is a Ser/Thr protein kinase that belongs to the phosphoinositide 3-kinase (PI3K)-related kinase (PIKK) family [1]. TOR is comprised of a cluster of amino-terminal HEAT repeats (Huntingtin, Elongation factor 3, A subunit of protein phosphatase $2 \mathrm{~A}$ and TOR1), a FAT domain (FRAP, ATM and TRRAP), a FKBP12-rapamycin binding (FRB) domain, a Ser/Thr kinase catalytic domain, and a FATC domain (FAT C-terminal). TOR exists in two functionally distinct multi-protein complexes named TOR complex 1 (TORC1) and TOR complex 2 (TORC2). In mammals, two accessory proteins named regulatory-associated protein of mTOR (RAPTOR) and rapamycin-insensitive companion of mTOR (RICTOR) distinguish TORC1 from TORC2, respectively $[2,3]$. Rapamycin and the $12-\mathrm{kDa}$ FK506-binding protein (FKBP12) form a complex that binds to and inhibits

\section{KARGER}

Fax +41613061234

E-Mail karger@karger.ch

www.karger.com
(C) 2012 S. Karger AG, Basel

0304-324X/12/0586-0524\$38.00/0

Accessible online at:

www.karger.com/ger
Yong Pan, $\mathrm{PhD}$

Gladstone Institute of Virology and Immunology

1650 Owens St, San Francisco, CA 94158 (USA)

Tel. +1 4157344971

E-Mail yong.pan@gladstone.ucsf.edu 
Fig. 1. A simplified model of TORC1-mediated regulation of the metabolome, $\mathrm{mi}$ tochondrial function and lifespan. The green ovals designate TORC1 signaling components activated by nutrients and insulin. The red ovals designate TORC1 signaling components suppressed by nutrients. The rectangles indicate the downstream physiological consequences of altered TORC1 signaling. Rapamycin and upstream signaling components like the Tsc proteins modulate TORC1 activity. Active TORC1 signals through downstream effectors, such as S6K, 4EBP and Atgs, causing alterations in metabolism and mitochondrial functions. These alterations result in changes in metabolomic profiles and ROS levels, which in turn impacts lifespan.

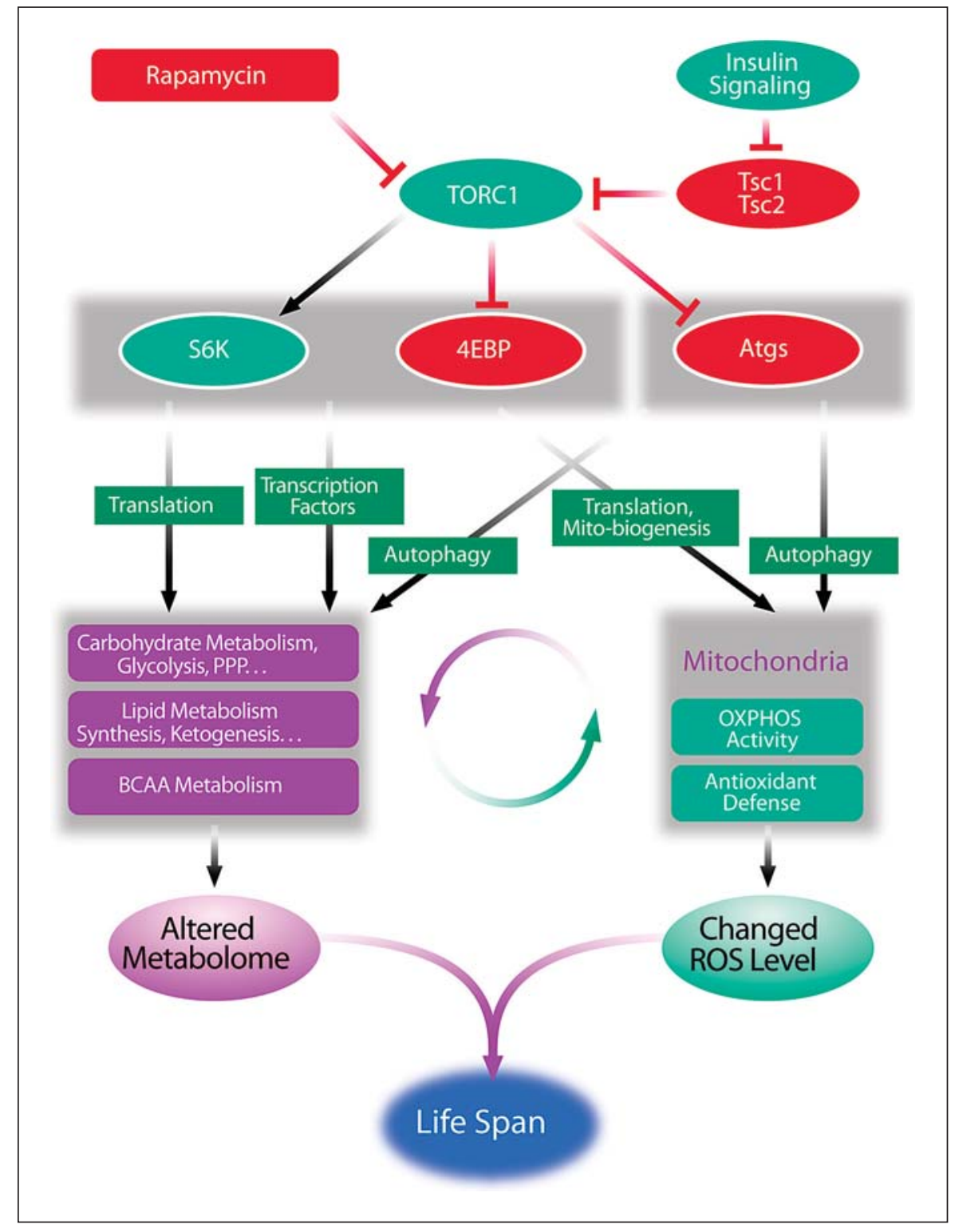

RAPTOR-bound TORC1, but not RICTOR-bound TORC2 [4]. While TORC2 has critical regulatory functions in cytoskeletal polarity and cell survival [3], TORC1 is the major nutrient sensor and therefore the subject of this mini-review. TORC1 lies at the intersecting point of diverse cellular inputs that include hormones (insulin or insulin-like growth factor 1, IGF-1), nutrients (glucose and amino acid), energy (ATP levels) and stress (reactive oxygen species). In mammals and flies, the tuberous sclerosis complex (Tsc1-Tsc2) integrates hormonal signals, and negatively regulates mammalian TORC1 (mTORC1) and fly TORC1, respectively (fig. 1). IGF-1 and insulin signal to mTORC1 via the PI3K-Akt pathway, which phosphorylates Tsc2. Phosphorylated Tsc2 dissociates from Tsc1 resulting in mTORC1 signaling [5].

Key downstream mediators of TORC1 signaling are also conserved among eukaryotic organisms. These include S6 kinase (S6K), the translation inhibitory factor $4 \mathrm{EBP}$ and several autophagy-related genes (Atgs). S6K is an AGC (protein kinase A, G and C) family kinase. Mammalian S6K as well as its orthologs in fly (dS6K) and in yeast (Sch9) are all directly phosphorylated and activated by TORC1 [6-8]. S6K phosphorylates a wide range of targets including ribosomal protein S6, thereby enhancing 
protein translation in the cytoplasm. In contrast, TORC1 phosphorylation of 4EBP disrupts its association with the translation factor eIF4E $[6,9,10]$. The release of eIF4E not only promotes global translation, but also preferentially enhances the translation of mRNAs with more complex 5' UTR structures. TORC1 also negatively regulates autophagy, while inhibition of TORC1 activity by genetic deletion or by rapamycin exposure induces autophagy. In yeast, Atg13 is hyperphosphorylated by TORC1 under nutrient-rich conditions, but is rapidly dephosphorylated after nitrogen starvation or rapamycin treatment [11]. Hypophosphorylated Atg13 then interacts with Atg1 and Atg17, thereby activating the kinase activity of Atg1 and initiating autophagy [11]. The ULK1-Atg13-FIP200 complex represents the mammalian ortholog of the yeast Atg1-Atg13-Atg17 complex, and is regulated by mTORC1 in a similar manner [12].

\section{Reduced TOR Activity Is Associated with Increased Lifespan}

There is growing evidence that the TORC1 pathway also regulates lifespan in mammals. Dietary supplementation of rapamycin at various growth stages extends lifespan in mice. Remarkably, initiation of rapamycin feeding late in life (day 600) in mice extends average lifespan without a change in body weight [13]. Treatment with rapamycin prolongs mean lifespan in both male and female mice by 9 and 13\%, respectively. Rapamycin feeding also significantly extends maximum lifespan, as shown by the proportion of treated mice living beyond the 90th percentile of mortality observed in the control population $(5.9 \%$ of the controls vs. $20.2 \%$ of the rapamycin-treated males, and $4.8 \%$ of the control vs. $21.5 \%$ of the rapamycin-treated females) [13]. Genetic models of reduced TORC1 signaling recapitulate these results. For example, female mice heterozygous for both mTOR and mLst8 $\left(\right.$ mtor $\left.^{+/-} \mathrm{mlst}^{+/-}\right)$exhibit an increase in mean lifespan by $14.4 \%$ relative to their wild-type counterparts [14]. The heterozygous males, surprisingly, do not exhibit longer lifespan with reduced TORC1 signaling [14]. TORC1 signaling also modulates lifespan in other genetic models. The Ames dwarf mouse, a long-lived mutant line with pituitary hormone deficiencies, shows reduced TORC1-mediated signaling levels in liver and muscle [15].

TORC1 controls lifespan in invertebrates as well as in eukaryotic microorganisms. Rapamycin feeding extends lifespan in several lines of D. melanogaster [16]. Expres- sion of a dominant negative form of TOR or overexpression of Tsc1 or Tsc2, both upstream inhibitors of TORC1, also extends fly lifespan [17]. In yeast, TORC1 regulates both replicative lifespan (as measured by the number of budding events by a given mother cell) and chronological lifespan (as measured by the duration of post-mitotic culture in stationary phase) [18-20]. Rapamycin treatment of yeast during various growth stages extends lifespan, as does reduction of TORC1 in mutants like the TOR1knockout yeast strain (tor $1 \Delta$ ) [18-20].

TORCl's effects on lifespan across species are mediated by several of its downstream effectors. Deletion of S6K1, an isoform of mammalian S6K, extends the lifespan of mice, particularly in females [21]. In flies, overexpression of a dominant negative form of Drosophila S6K (dS6K) increases lifespan, while the constitutively active form decreases lifespan [17]. In yeast, deletion of the S6K ortholog $\mathrm{SCH} 9$ extends both replicative and chronological lifespan $[18,22]$. Because the knockout of many mTORC1 signaling components results in embryonic lethality, epistasis analyses have been primarily attempted in flies and yeast. Overexpression of S6K or suppression of 4EBP negate the lifespan extension induced by rapamycin treatment [16]. Similarly, yeast sch 9 knockout (sch9 9 ) extends lifespan, but the tor1-sch9 double knockout (tor1 $\Delta$ sch9 9 ) does not prolong lifespan beyond what is observed in the sch $9 \Delta$ mutant alone [23]. These observations show that suppression of cytoplasmic protein translation is a conserved mechanism by which TORC1 prolongs lifespan [10, 24]. Probing the effects of translation in mammalian lifespan continues to be a challenge, but results from mice lacking the translational regulator S6K1 are consistent with experimental data from yeast and flies [21]. Regulation of autophagy is also critical in TORC1 regulation of lifespan. Rapamycin-induced lifespan extension in yeast and flies is abrogated if autophagy is inhibited [16, 25]. Multiple autophagy-defective yeast mutants are short-lived, a phenotype that cannot be rescued by rapamycin [25]. Similarly, downregulation of Atg5 reduces the lifespan of the fruit fly treated with rapamycin [16].

In contrast to the global knockout of TORC1 signaling components, tissue-specific knockouts yield intriguing insights into how the local regulation of TORC1 signaling can impact global physiology and lifespan. In flies, expression of the dominant negative form of dTOR or dS6K, or overexpression of dTsc 2 in fat tissue alone, is sufficient to increase lifespan [17]. In mice, fat-specific deletion of RAPTOR expression causes a global increase in insulin sensitivity and protects against weight gain (in mice fed either a high-fat diet or normal chow), which are two 
common aging-associated metabolic abnormalities [26]. While a high-fat diet decreases insulin sensitivity, induces S6K hyperphosphorylation and AKT hypophosphorylation, RAPTOR knockout restores the loss of insulininduced AKT phosphorylation [26]. No aging study has yet been reported on mice with a fat-specific reduction of TORC1, but mice with reduced insulin signaling via fatspecific knockout of the insulin receptor (FIRKO mice) do live longer [27]. Interestingly, FIRKO and fat-specific RAPTOR knockout mice both exhibit lower body weight, lower fat mass and better glucose tolerance compared to wild-type controls, even though their fat tissues do not respond to insulin. In contrast, skeletal muscle-specific knockout of RAPTOR (RAmKO) shortens lifespan [28]. Compared to controls, RAmKO mice develop kyphosis, muscle dystrophy and other aging-related phenotypes, thereby revealing a tissue-specific role for TORC1 in lifespan regulation [28].

TORC1 has been implicated as a key mediator of the effects of dietary restriction (DR). DR, defined as a decrease in calorie intake (10-50\%) without malnutrition, increases lifespan in multiple species from yeast to mammals [29]. Importantly, in yeast and fruit flies, DR does not increase the lifespan of organisms carrying a TOR deletion $[17,18]$. DR also extends lifespan in wild-type mice, and their gene expression profiles resemble those of S6K1-knockout mice, suggesting that DR acts through mTORC1 inhibition [21]. While the evidence supporting a role for TORC1 in DR-induced lifespan extension is compelling, caveats must also be noted. First, it still remains unclear whether tissue-specific TORC1 signaling is sufficient, or whether global TORC1 downregulation is required for the beneficial effects of DR. Whether and how TORC1 interacts with other known lifespan modulators like the sirtuins remains unknown. Furthermore, interactions between TORC1 and DR may be regulated by genetic background, as DR has drastically different effects on lifespan in different mouse strains [30].

\section{Metabolome: You Are Not Only What You Eat}

Metabolomic analysis, defined as the unbiased measurement of a large number of cellular metabolites, revealed that active TORC1 stimulates carbohydrate metabolism and that this effect is suppressed by rapamycin [31]. Knockout of the negative TORC1 regulator Tsc2 $\left(\mathrm{T}^{-/-}\right)$leads to TORC1 activation and the upregulation of gene expression for glycolytic and oxidative pentose phosphate proteins, resulting in accumulation of the in-

Metabolic Regulation, Mitochondria and the Life-Prolonging Effect of Rapamycin termediate metabolites glucose-6-phosphate, dihydroxyacetone phosphate, lactate and ribose-5-phosphate [31]. TORC1 signals to several carbohydrate metabolic pathways: S6K activation signals to increase transcription of the oxidative arm of the pentose phosphate pathway, while 4EBP modulates glycolysis via translational control of the hypoxia-inducible factor $1 \alpha(\mathrm{Hif} 1 \alpha)$ [31].

TORC1 also regulates lipid metabolism and ketogenesis. TORC1 increases de novo lipid synthesis in a S6Kdependent fashion [31], and suppresses lipid breakdown and ketogenesis. Under fasting conditions, TORC1 activity is decreased and total serum ketone levels are increased [32]. Constitutively active hepatic TORC1 in the absence of Tscl attenuates fasting-induced ketogenesis: lipid breakdown and total ketones are both reduced in the liver [32]. Cell culture studies confirm that the knockdown of Tscl suppresses ketogenesis, which is restored by rapamycin treatment [32].

TORC1 signaling is implicated in amino acid metabolism. Branched chain amino acids (BCAAs), i.e. leucine, isoleucine and valine, induce the phosphorylation of mTOR and activation of the TORC1 pathway in mice and rats $[33,34]$. A high-fat diet combined with BCAAs induces insulin resistance, which is reversed by rapamycin treatment [33]. Unexpectedly, feeding dietary BCAAs alone to middle-aged mice is associated with increased median lifespan, although not maximum lifespan [34]. The mechanism responsible for these effects is poorly understood, but these results highlight the importance of defining the effect of TORC1 on lifespan in a tissue-specific context. Intriguingly, BCAAs activate TORC1-regulated mitochondrial biogenesis selectively in skeletal muscle and not in fat or liver [34]. Therefore, the beneficial effects of BCAAs are likely to be mediated in a celltype-specific manner by active muscle TORC1 signaling.

How TORC1-induced metabolomic alterations influence aging is still poorly understood, but it is likely that both autophagy and translation control represent two critical pathways. Autophagy, which is negatively regulated by TORC1, plays a critical role in amino acid homeostasis. Plasma amino acid concentrations, especially for the essential amino acids and BCAAs, rapidly decrease in autophagy-defective compared to wild-type mice [35]. In wild-type yeast cultured in nitrogen-deficient media, amino acid pools initially decrease but are partially replenished thereafter. By comparison, in autophagy mutants this replenishment does not occur, indicating that TORC1-regulated autophagy is critical for maintaining cellular viability during nitrogen starvation [36]. Autophagy also plays a role in lipid metabolism: 
macrolipophagy, defined as the sequestration of cellular lipid droplets inside autophagosomes for lysosomal degradation, releases free fatty acids for mitochondrial $\beta$-oxidation and energy production [37]. Inhibition of autophagy by TORC1 in hepatocytes leads to suppressed $\beta$-oxidation and lipid accumulation [37]. Like autophagy, protein translation is closely associated with nutrient availability. Suppressed translation signals amino acid biosynthesis regulator Gcn4, which is required for lifespan extension in tor $1 \Delta$ or $\operatorname{sch} 9 \Delta$ [24]. Therefore, by sensing the abundance of various nutrients and regulating the activity of critical processes such as autophagy and translation, the TORC1 signaling pathway lies at the intersection between environmental and innate mechanisms of aging.

\section{Mitochondria: An Intersection for TORC1 and Lifespan}

Mitochondria play key roles in energy production, intermediary metabolism and cellular signaling. They contain enzymes for critical metabolic pathways, including the tricarboxylic acid (TCA) cycle, fatty acid $\beta$-oxidation and oxidative phosphorylation (OXPHOS). OXPHOS is the major cellular source of ATP, reactive oxygen species (ROS), reactive nitrogen species (RNS) and other strong oxidants. To counteract the damage to macromolecules and cellular components caused by reactive species, especially ROS, mitochondria deploy antioxidant defense mechanisms such as superoxide dismutase 2 (SOD2). Denham Harman first proposed that the accumulation of mitochondrial ROS induces cellular damage and thereby decrease lifespan. Harman's simple emphasis on the damaging effects of mitochondrial ROS to cells and the negative impact on lifespan has since been challenged by many studies [reviewed in 38]. Not recognized by Harman's initial theory, mitochondrial ROS significantly influence cellular signaling by activating key metabolic regulators such as Hif- $1 \alpha$ [reviewed in 39]. Although the current understanding of the mitochondrial impact on lifespan is far from perfect, a growing body of evidence supports the concept that mitochondrial function critically influences lifespan through its effects on OXPHOS activity and ROS levels.

Importantly, TORC1 signaling regulates mitochondrial biogenesis and turnover in mammals as well as lower model organisms. In mice, the effect of TORC1 on mitochondrial function heavily depends on the tissue being studied. In skeletal muscle, rapamycin reduces the ex- pression of genes encoding OXPHOS components in a PGC1 $\alpha$-dependent fashion [40]. In agreement with these observations, RAmKO mice show lower OXPHOS levels and abnormal mitochondrial biogenesis [28], while TORC1 activation by BCAAs in muscle cells promotes mitochondrial biogenesis [34]. In contrast, mice with fatty tissue-specific knockout of RAPTOR expression have higher rates of respiration [26]. This protects the mice against weight gain on both regular and high-fat diets because increased mitochondrial uncoupling results in higher energy expenditure [26]. FIRKO mice, like the RAPTOR knockout mice, exhibit increased respiration and higher energy expenditure because they have an elevated expression of genes involved in OXPHOS and $\beta$ oxidation [41]. Intriguingly, the greatest difference in OXPHOS expression occurs in older mice [41]. The inverse relationship between TORC1 activity and respiration in fat tissues is similar to what is observed in yeast, whereby rapamycin treatment or deletion of TORC1 components increases oxygen consumption [20]. The expression of proteins from respiratory complexes, encoded by both the nuclear and mitochondrial genomes, is globally upregulated [23].

Downstream of TORC1, S6K, 4EBP and Atg-family proteins all modulate mitochondrial biogenesis. Gene expression of PGC1 $\alpha$ - and AMPK-regulated pathways is upregulated in the liver, muscle or white adipose tissue of S6K knockout mice [21]. However, the mechanism underlying these changes is not clear, while mRNAs encoding mitochondrial components do not significantly increase, and translational control may be involved, similar to what is observed for 4EBP (discussed below). In contrast to mammalian S6K, the role of the yeast ortholog Sch9 in regulating OXPHOS is better defined. Sch9 is a critical downstream effector of TOR and is involved in regulating mitochondrial oxygen consumption. Like tor $1 \Delta$, sch $9 \Delta$ strains show increased levels of nuclear and mitochondria-encoded OXPHOS proteins [23]. In fruit flies, 4EBP is a major translational regulator of OXPHOS components. DR alters the mRNA translational profile in a 4EBP-dependent fashion [10]. mRNAs encoding OXPHOS proteins possess a short 5' UTR and weak secondary structure. Translation of OXPHOS-encoding mRNA is therefore less dependent on eIF4E than translation of other mRNA species. Consequently, the expression of OXPHOS is maintained during DR despite reductions in global translation [10]. TORC1 not only regulates mitochondrial biogenesis through S6K and 4EBP, but also regulates mitochondrial turnover through the Atgs. Asymmetrical fission separates functional and dysfunc- 
tional mitochondria, which are subsequently degraded by autophagy [42]. Autophagy therefore participates in mitochondria 'quality control'.

As a consequence of its ability to regulate mitochondrial biogenesis and turnover, TORC1 influences the levels of mitochondrial ROS. Defects in the clearance of damaged mitochondria by TORC1-regulated autophagy also contribute to ROS accumulation [43]. Isolated mitochondria obtained from mouse skeletal muscle lacking Atg7 $\left(\right.$ Atg $\left.7^{-/-}\right)$exhibit a significant defect in mitochondrial respiration as well as increased levels of ROS [43]. The positive correlation between TORC1 signaling and ROS is also observed in lower organisms. In yeast, reduced oxidative damage was observed in the stationary phase culture of tor $1 \Delta$ and $\operatorname{sch} 9 \Delta$ strains $[20,22]$. Interestingly, both rapamycin treatment and torl knockout in yeast cause a temporary increase in superoxide levels during the log growth phase because these manipulations promote OXPHOS biogenesis [20]. A transient peak of mitochondrial ROS likely serves as a hormetic stimulus, which induces the expression of proteins involved in oxidative stress resistance and thereby extends lifespan [reviewed in 39]. This data raises the intriguing possibility that mitochondrial hormesis (or mitohormesis) mediates the effect of TORC1 on lifespan in mammals and in lower organisms.

It is therefore likely that mitochondrial OXPHOS function and changes in oxidative stress responses collaborate with metabolomic alterations to regulate lifespan (fig. 1). TCA cycle intermediates, such as $\alpha$-ketoglutarate and mitochondrial ROS, regulate the activity of transcription factors such as Hifl $\alpha$ to change metabolism [reviewed in 39]. On the other hand, key metabolic pathways also influence ROS levels. For example, BCAAs reduce tissue hydrogen peroxide and oxidative damage levels and increase antioxidant defense signaling [34]. Although BCAAs induce TORC1 signaling in both liver and muscle [33], only active TORC1 enhances mitochondrial biogenesis and antioxidant defense mechanisms in muscle. Therefore, tissue-specific signaling processes heavily regulate the interactions between metabolome and mitochondrial activities, thereby influencing lifespan.

\section{Aging: Above and beyond Rapamycin}

Numerous studies have documented the role of rapamycin and TORC1 signaling in the determination of lifespan in various organisms. TORC1 signaling influences lifespan via its ability to regulate both metabolic pathways and oxidative damage (fig. 1). Such pathways are non-linear, since different signaling components and phenotypic outputs interact with each other in a network. Manipulation of TORC1 activity, either via DR or rapamycin treatment, can regulate lifespan. Much remains to be learned about the molecular mechanisms of these important effects. While there is no clinical evidence showing that rapamycin extends human lifespan, the evolutionary conservation of the TORC1 pathway and its effects in murine models warrant further study of this pathway in the control of human lifespan.

\section{Acknowledgements}

Dr. Y. Nishida is supported by Japanese Society for the Promotion of Science. The authors would like to acknowledge Drs. Wenjuan He and Anna Lisa Lucido for editorial assistance and constructive suggestions. The authors also thank John Carroll for figure preparation.

\section{References}

$>1$ Brunn GJ, Hudson CC, Sekulic A, Williams JM, Hosoi H, Houghton PJ, Lawrence JC Jr, Abraham RT: Phosphorylation of the translational repressor PHAS-I by the mammalian target of rapamycin. Science 1997;277: 99-101.

-2 Hara K, Maruki Y, Long X, Yoshino K, Oshiro N, Hidayat S, Tokunaga C, Avruch J, Yonezawa K: Raptor, a binding partner of target of rapamycin (TOR), mediates tor action. Cell 2002;110:177-189.

$>3$ Sarbassov DD, Ali SM, Kim DH, Guertin DA, Latek RR, Erdjument-Bromage $\mathrm{H}$, Tempst P, Sabatini DM: Rictor, a novel bind- ing partner of mTOR, defines a rapamycininsensitive and raptor-independent pathway that regulates the cytoskeleton. Curr Biol 2004; 14:1296-1302.

4 Yip CK, Murata K, Walz T, Sabatini DM, Kang SA: Structure of the human mTOR complex I and its implications for rapamycin inhibition. Mol Cell 2010;38:768-774.

$\checkmark 5$ Inoki K, Li Y, Zhu T, Wu J, Guan KL: TSC2 is phosphorylated and inhibited by Akt and suppresses mTOR signalling. Nat Cell Biol 2002;4:648-657

6 Burnett PE, Barrow RK, Cohen NA, Snyder SH, Sabatini DM: RAFT1 phosphorylation of the translational regulators p70 S6 kinase and 4E-BP1. Proc Natl Acad Sci USA 1998; 95:1432-1437.

7 Urban J, Soulard A, Huber A, Lippman S, Mukhopadhyay D, Deloche O, Wanke V, Anrather D, Ammerer G, Riezman H, Broach JR, De Virgilio C, Hall MN, Loewith R: Sch9 is a major target of TORC1 in Saccharomyces cerevisiae. Mol Cell 2007;26:663-674.

$\checkmark 8$ Watson KL, Chou MM, Blenis J, Gelbart WM, Erikson RL: A Drosophila gene structurally and functionally homologous to the mammalian 70-kDa S6 kinase gene. Proc Natl Acad Sci USA 1996;93:13694-13698. 
9 Beretta L, Gingras AC, Svitkin YV, Hall MN, Sonenberg N: Rapamycin blocks the phosphorylation of 4E-BP1 and inhibits cap-dependent initiation of translation. EMBO J 1996;15:658-664.

-10 Zid BM, Rogers AN, Katewa SD, Vargas MA, Kolipinski MC, Lu TA, Benzer S, Kapahi P: 4E-BP extends lifespan upon dietary restriction by enhancing mitochondrial activity in Drosophila. Cell 2009;139:149-160.

- 11 Kamada Y, Funakoshi T, Shintani T, Nagano $\mathrm{K}$, Ohsumi M, Ohsumi Y: Tor-mediated induction of autophagy via an Apg1 protein kinase complex. J Cell Biol 2000;150:15071513.

-12 Hosokawa N, Hara T, Kaizuka T, Kishi C, Takamura A, Miura Y, Iemura S, Natsume T, Takehana K, Yamada N, Guan JL, Oshiro N, Mizushima N:Nutrient-dependentmTORC1 association with the ULK1-Atg13-FIP200 complex required for autophagy. Mol Biol Cell 2009;20:1981-1991.

-13 Harrison DE, Strong R, Sharp ZD, Nelson JF, Astle CM, Flurkey K, Nadon NL, Wilkinson JE, Frenkel K, Carter CS, Pahor M, Javors MA, Fernandez E, Miller RA: Rapamycin fed late in life extends lifespan in genetically heterogeneous mice. Nature 2009;460:392-395.

14 Lamming DW, Ye L, Katajisto P, Goncalves MD, Saitoh M, Stevens DM, Davis JG, Salmon $\mathrm{AB}$, Richardson A, Ahima RS, Guertin DA, Sabatini DM, Baur JA: Rapamycin-induced insulin resistance is mediated by mTORC2 loss and uncoupled from longevity. Science 2012;335:1638-1643.

15 Sharp ZD, Bartke A: Evidence for down-regulation of phosphoinositide 3-kinase/Akt/ mammalian target of rapamycin (PI3K/Akt/ mTOR)-dependent translation regulatory signaling pathways in Ames dwarf mice. J Gerontol A Biol Sci Med Sci 2005;60:293300.

16 Bjedov I, Toivonen JM, Kerr F, Slack C, Jacobson J, Foley A, Partridge L: Mechanisms of lifespan extension by rapamycin in the fruit fly Drosophila melanogaster. Cell Metab 2010;11:35-46.

$\checkmark 17$ Kapahi P, Zid BM, Harper T, Koslover D, Sapin V, Benzer S: Regulation of lifespan in Drosophila by modulation of genes in the TOR signaling pathway. Curr Biol 2004; 14 : 885-890.

18 Kaeberlein M, Powers RW 3rd, Steffen KK, Westman EA, Hu D, Dang N, Kerr EO, Kirkland KT, Fields S, Kennedy BK: Regulation of yeast replicative life span by TOR and Sch 9 in response to nutrients. Science $2005 ; 310$ : 1193-1196.

-19 Powers RW 3rd, Kaeberlein M, Caldwell SD, Kennedy BK, Fields S: Extension of chronological lifespan in yeast by decreased TOR pathway signaling. Genes Dev 2006;20:174184.

20 Pan Y, Schroeder EA, Ocampo A, Barrientos A, Shadel GS: Regulation of yeast chronological lifespan by TORC1 via adaptive mito- chondrial ROS signaling. Cell Metab 2011; 13:668-678.

21 Selman C, Tullet JM, Wieser D, Irvine E, Lingard SJ, Choudhury AI, Claret M, Al-Qassab $\mathrm{H}$, Carmignac D, Ramadani F, Woods A, Robinson IC, Schuster E, Batterham RL, Kozma SC, Thomas G, Carling D, Okkenhaug K, Thornton JM, Partridge L, Gems D, Withers DJ: Ribosomal protein S6 kinase 1 signaling regulates mammalian lifespan. Science 2009;326:140-144.

22 Fabrizio P, Pozza F, Pletcher SD, Gendron $\mathrm{CM}$, Longo VD: Regulation of longevity and stress resistance by $\mathrm{Sch} 9$ in yeast. Science 2001:292:288-290.

23 Pan Y, Shadel GS: Extension of chronological life span by reduced TOR signaling requires down-regulation of Sch9p and involves increased mitochondrial OXPHOS complex density. Aging (Albany NY) 2009;1:131-145.

24 Steffen KK, MacKay VL, Kerr EO, Tsuchiya M, Hu D, Fox LA, Dang N, Johnston ED, Oakes JA, Tchao BN, Pak DN, Fields S, Kennedy BK, Kaeberlein M: Yeast life span extension by depletion of 60 s ribosomal subunits is mediated by Gcn4. Cell 2008;133: 292-302.

25 Alvers AL, Wood MS, Hu D, Kaywell AC, Dunn WA, Aris JP: Autophagy is required for extension of yeast chronological lifespan by rapamycin. Autophagy 2009;5:847-849.

26 Polak P, Cybulski N, Feige JN, Auwerx J, Ruegg MA, Hall MN: Adipose-specific knockout of raptor results in lean mice with enhanced mitochondrial respiration. Cell Metab 2008;8:399-410.

27 Bluher M, Kahn BB, Kahn CR: Extended longevity in mice lacking the insulin receptor in adipose tissue. Science 2003;299:572-574.

28 Bentzinger CF, Romanino K, Cloetta D, Lin S, Mascarenhas JB, Oliveri F, Xia J, Casanova E, Costa CF, Brink M, Zorzato F, Hall MN, Ruegg MA: Skeletal muscle-specific ablation of raptor, but not of rictor, causes metabolic changes and results in muscle dystrophy. Cell Metab 2008;8:411-424.

29 Mair W, Dillin A: Aging and survival: the genetics of lifespan extension by dietary restriction. Annu Rev Biochem 2008;77:727754.

30 Liao CY, Rikke BA, Johnson TE, Diaz V, Nelson JF: Genetic variation in the murine lifespan response to dietary restriction: from life extension to life shortening. Aging Cell 2010;9:92-95.

31 Duvel K, Yecies JL, Menon S, Raman P, Lipovsky AI, Souza AL, Triantafellow E, Ma Q, Gorski R, Cleaver S, Vander Heiden MG, MacKeigan JP, Finan PM, Clish CB, Murphy LO, Manning BD: Activation of a metabolic gene regulatory network downstream of mTOR complex 1. Mol Cell 2010;39:171-183.

- 32 Sengupta S, Peterson TR, Laplante M, Oh S, Sabatini DM: mTORC1 controls fasting-induced ketogenesis and its modulation by ageing. Nature 2010;468:1100-1104.
33 Newgard CB, An J, Bain JR, Muehlbauer MJ, Stevens RD, Lien LF, Haqq AM, Shah SH, Arlotto M, Slentz CA, Rochon J, Gallup D, Ilkayeva O, Wenner BR, Yancy WS Jr, Eisenson $H$, Musante G, Surwit RS, Millington DS, Butler MD, Svetkey LP: A branchedchain amino acid-related metabolic signature that differentiates obese and lean humans and contributes to insulin resistance. Cell Metab 2009;9:311-326.

34 D’Antona G, Ragni M, Cardile A, Tedesco L, Dossena M, Bruttini F, Caliaro F, Corsetti G, Bottinelli R, Carruba MO, Valerio A, Nisoli E: Branched-chain amino acid supplementation promotes survival and supports cardiac and skeletal muscle mitochondrial biogenesis in middle-aged mice. Cell Metab 2010;12: 362-372.

35 Komatsu M, Waguri S, Ueno T, Iwata J, Murata S, Tanida I, Ezaki J, Mizushima N, Ohsumi Y, Uchiyama Y, Kominami E, Tanaka K, Chiba T: Impairment of starvation-induced and constitutive autophagy in Atg7-deficient mice. J Cell Biol 2005; 169: 425-434.

36 Onodera J, Ohsumi Y: Autophagy is required for maintenance of amino acid levels and protein synthesis under nitrogen starvation. J Biol Chem 2005;280:31582-31586.

37 Singh R, Kaushik S, Wang Y, Xiang Y, Novak I, Komatsu M, Tanaka K, Cuervo AM, Czaja MJ: Autophagy regulates lipid metabolism. Nature 2009;458:1131-1135.

38 Jang YC, Remmen VH: The mitochondrial theory of aging: Insight from transgenic and knockout mouse models. Exp Gerontol 2009;44:256-260.

-39 Pan Y: Mitochondria, reactive oxygen species, and chronological aging: a message from yeast. Exp Gerontol 2011;46:847-852.

40 Cunningham JT, Rodgers JT, Arlow DH, Vazquez F, Mootha VK, Puigserver P: mTOR controls mitochondrial oxidative function through a YY1-PGC-1 $\alpha$ transcriptional complex. Nature 2007;450:736-740.

41 Katic M, Kennedy AR, Leykin I, Norris A, McGettrick A, Gesta S, Russell SJ, Bluher M, Maratos-Flier E, Kahn CR: Mitochondrial gene expression and increased oxidative metabolism: role in increased lifespan of fatspecific insulin receptor knockout mice. Aging Cell 2007;6:827-839.

-42 Twig G, Elorza A, Molina AJ, Mohamed H, Wikstrom JD, Walzer G, Stiles L, Haigh SE, Katz S, Las G, Alroy J, Wu M, Py BF, Yuan J, Deeney JT, Corkey BE, Shirihai OS: Fission and selective fusion govern mitochondrial segregation and elimination by autophagy. EMBO J 2008;27:433-446.

43 Wu JJ, Quijano C, Chen E, Liu H, Cao L, Fergusson MM, Rovira II, Gutkind S, Daniels MP, Komatsu M, Finkel T: Mitochondrial dysfunction and oxidative stress mediate the physiological impairment induced by the disruption of autophagy. Aging (Albany NY) 2009;1:425-437. 